\title{
MEN: Multi-Attribute Feature Selection for Face Recognition
}

\author{
P. Ramaraj \\ Research Scholar, Assistant Professor, \\ Department of Computer Science, \\ Government Arts College, Ariyalur, \\ Tamilnadu, India.
}

\author{
M. Prabakaran \\ Research Advisor Assistant Professor, \\ Department of Computer Science, \\ Government Arts College, Ariyalur, \\ Tamilnadu, India.
}

\begin{abstract}
Face recognition has become more sophisticated in biometric based authentication systems, which uses various facial features. The authentication mechanism is still required with more features to be used and has to be done in a short time. Existing face recognition algorithms are more scalable in time and memory used, also produces high frequency of false positive results. To overcome the problem of false positive results, we propose MEN-Mouth, Eye and Nose features based multi attribute feature selection method for face recognition. The proposed method extracts the facial features like Nose, mouth and eye, from extracted features we compute eccentric measures for each of the feature. The eccentric measure is computed between four axis co-ordinates of facial features. Computed features are converted into single feature, and computes feature weight based on computed feature set. The computed feature weight is used to recognize the person.
\end{abstract}

\section{Index Terms}

Facial Features, Face Recognition, Multi Attribute, Bio medical Features.

\section{INTRODUCTION}

Face recognition an unavoidable process in various domains where the human access to any resource has to be restricted. For example, face recognition has been used in defense sector, banking and many more. In case of defense, the access to weapon store can be restricted by means of bio metric authentication, and facial features can be used as an authentication source. The face features like eyes, nose and mouth could be used as authentication material. Presently the face recognition has been used mostly to overcome the hackers, crackers and un authorized activities in the world. There have been so many biometric methods and system is available like Iris scanning, for example, is very reliable but too intrusive; fingerprints are socially accepted, but not applicable to nonconsentient people. On the other hand, face recognition represents a good compromise between what's socially acceptable and what's reliable, even when operating under controlled conditions. In last decade, many algorithms based on linear/nonlinear methods, neural networks, wavelets, etc. have been proposed. Nevertheless, Face Recognition Vendor Test 2002 shown that most of these approaches encountered problems in outdoor conditions. This lowered their reliability compared to state of the art biometrics. What exactly faces recognition is Face recognition technology is the least intrusive and fastest biometric technology.

The modern face detectors are mostly appearance-based method, which means that they need training data to learn the classifiers. Collecting a large amount of ground truth data remains a very expensive task, which certainly demands more research. Schemes such as multiple instance learning boosting and multiple category boosting are helpful in reducing the accuracy needed for the labeled data, though ideally one would like to leverage unlabeled data to facilitate learning. Unsupervised or semi-supervised learning schemes would be very ideal to reduce the amount of work needed for data collection.

Biometric authentication has become popular in this era, where many biomedical features are used for person authentication and identification. Most of the banking sectors started using biometric features like face, finger print and palm prints. Human face has more features like eyes, mouth and nose, also the finger has prints which is an most popular feature varying between persons. The palm print, which shows the lines in hands and has different distance between lines for each person in the world. All of them has more impact on person authentication, we consider the facial features for person authentication and recognition purpose.

Facial features includes, Eyes, mouth, noses, where eye contains features like size of eye, width and height of eyes, which will be varying for each person. The mouth contains many features like width of mouth and height of lips, which can be used for authentication. The Nose has two features namely the height of nose and width of the nose. All these features are distinct and will vary from person to person which can be used for person authentication. We propose a new methodology which uses all these features we discussed and more than that we use a new eccentric measure, which will be calculated for each of the feature using their height and width. Finally a eccentric measure is computed using the co-ordinate of all the three facial feature to compute the feature weight to identify the person.

\section{BACKGROUND}

There are many methods has been discussed in the literature, we discuss few of them here for understanding the problem.

PCA based method discussed in [1] conducted survey on face recognition as it has received significant attention in recent years. After few decades of research it is able to provide promising solutions for the applications such as commercial and law enforcement. The availability of protocols viz., FERET, XM2VTS, and MPEG-7 has made researchers to improve algorithms with constraints. Face recognition system for Enhanced e-health [4] implemented a reliable PCA based face recognition system and evaluated the performance using standard face databases such as Indian database and the Face recognition data, University of Essex, UK. The different techniques such as sum of absolute difference, sum of squared difference and normalized cross correlation are used for matching unknown images with known images. 
Minimum distance classifier [3] proposed an efficient algorithm to detect human behaviors for visual surveillance. The face recognition technique is for dynamic scenario using PCA and minimum distance classifier. The mathematical analysis is made on the video of human face captured to understand or interpret the behavior. Appearance based face recognition [5] presented a Two-Dimensional Neighborhood Preserving Projection (2DNPP) for appearance based face representation and recognition. 2DNPP enables us to directly use a feature input of 2D image.

LBP based texture for face classification [6] conducted survey on local binary patterns. First several different approaches are compared, and then the best fusion approach is tested on different datasets and compared with several approaches. The experiments show that a fusion approach based on uniform Local Quinary Pattern (LQP) and a rotation invariant LQP, where a bin selection based on variance is performed and Neighborhood Preserving Embedding (NPE) feature transform is applied to obtain better results on six different datasets with support vector machine classifier.

Binary patterns for face recognition [7] proposed Principal Local Binary Patterns (PLBP) for recognizing faces. The attribute evaluator algorithm of the data mining tool Weka is used. It is assumed that each face region has a different influence on the recognition process; a 9-region mask is designed and a set of optimized weights are obtained for the mask by means of the data mining tool RapidMiner. The method is tested with the FERET database.

An Automatic Face Detection System for RGB Images[8] is proposed that works for digital color images. Our automatic detection method is based on image skin regions, therefore a skin-based segmentation of RGB images is provided first. Then, we decide for each skin region if it represents a human face or not, using a set of candidate criteria, an edge detection process, a correlation based technique and a threshold-based method. A high face detection rate is obtained using the proposed method.

DBC based Face Recognition using DWT [9], is proposed using face biometric has proved its reliability in last decade. In this ,they propose DBC based Face Recognition using DWT (DBC- FR) model. The Poly-U NearInfra Red (NIR) database images are scanned and cropped to get only the face part in preprocessing. The face part is resized to $100 * 100$ and DWT is applied to derive LL, LH, HL and HH subbands. The LL subband of size $50 * 50$ is converted into 100 cells with $5 * 5$ dimention of each cell. The Directional Binary Code (DBC) is applied on each $5^{*} 5$ cell to derive 100 features. The Euclidian distance measure is used to compare the features of test image and database images.

All the above mentioned methods has the problem of mismatching and false positive results. We propose a new face recognition algorithm which uses multi attribute based facial features with eccentric measures to recognize the faces.

\section{PROPOSED METHOD}

The proposed MEN-Mouth Eye Nose based multi attribute feature selection approach has the following stages namely: Feature Identification, Feature Extraction, Eccentric Measure Computation, Feature weight computation, and Finally Face recognition. At the first stage the face features are identified and in the second stage the identified features are extracted. At the third stage we compute the eccentric measure for each of the extracted features and in the fourth stage a feature weight is computed. Finally at the final stage face recognition is performed.

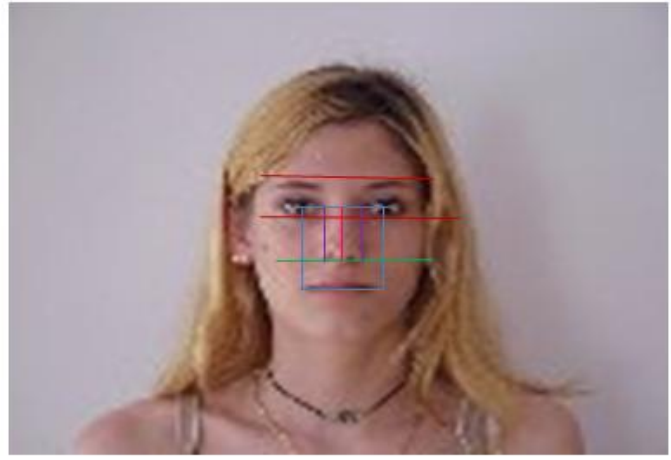

Figure1: Example view of features marked.

From figure1, the red lines shows the height and width of the eyes marked and extracted, whereas the blue lines shows the eccentric marks of four axis and components of the face. The green line shows the size of mouth and the violet line shows the size of nose marked.

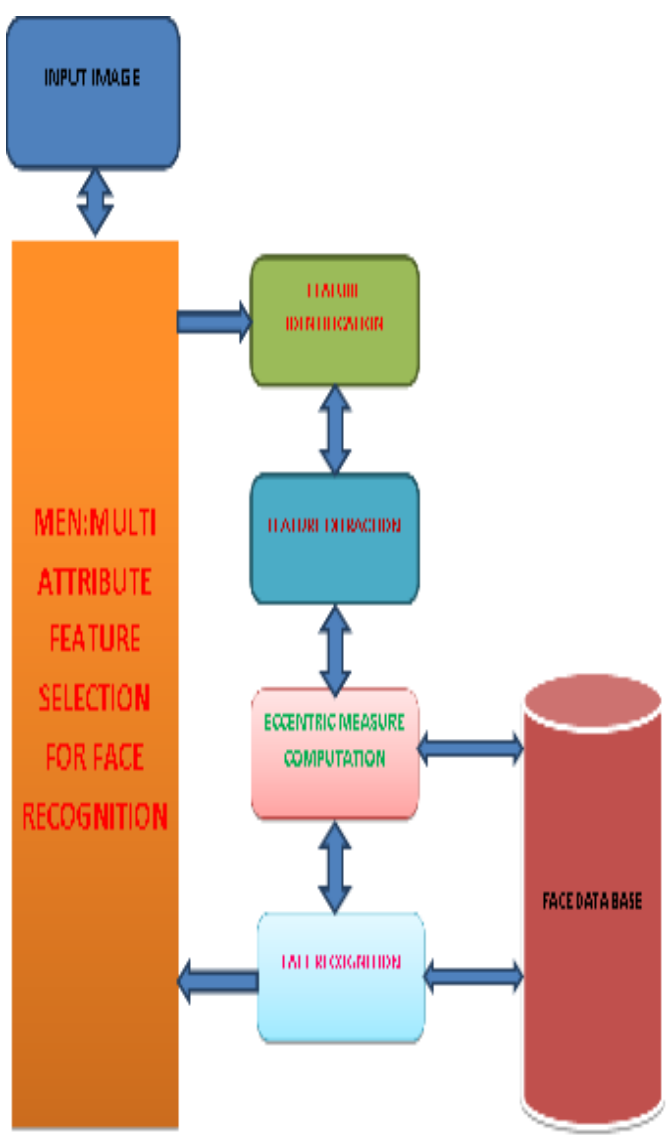

Figure2: Proposed method architecture

\subsection{Feature Identification}

At the first phase, facial features and their locations is identified using template matching techniques. The proposed method maintains features and their templates for each of the facial features. Each template is matched with the input face image to find out the location of the feature. For eyes, the template contains iris with eye brows are matched to find out the location of the feature. We perform identification of left and right eyes to identify the location of nose and its edges. Similarly the location of mouth feature is matched with available templates. 


\subsection{Feature Extraction}

With the identified location of each feature the following features are extracted for each of the facial components. For eyes, we extract the left eye region and computes the width and height of eye , similarly the edges of right eye is extracted and its height and width are computed. Then set of boundary points are computed to calculate the size of eye region. Now iris location is identified with the extracted eye and its midpoint is selected, which will be used for next level process. Similarly the features of nose as its height and width are extracted to be used in further stages of computing. The mouth features like lips height and width are computed. Further horizontal diagonal edge points of mouth and two centers of iris are connected to form a rectangle in order to compute the biometric square. The biometric square becomes an important key in this method which decides the recognition process to be successful. The computed biometric square has more impact in face recognition because the region affected will be different for every person.

\section{Algorithm:}

Step1: start

Step2: initialize feature vector Fv.

Step3: read face features identified FF.

Step4: Read left eye LE.

$$
\begin{gathered}
\text { Compute left eye width Lew } \\
\sqrt{(L E . X 2-L E . X 1)^{2}+(\text { LE.Y2-LE.Y1 })^{2}} \\
\text { Compute left eye height Leh }= \\
\int \operatorname{Dist(LE.TopBrow-LE.BottomBrow)}
\end{gathered}
$$$$
\text { compute left eye } \mathrm{X} \text {-axis start point LeX. }
$$

compute left eye y-axis start point LeY.

step5: Read right eye RE.

Compute left eye width Rew $=\sqrt{(R E . X 2-R E . X 1)^{2}}+($ RE.Y2RE.Y1 $)^{2}$

Compute right eye height Reh $=\int \operatorname{Dist}(R E$.TopBrow RE.BottomBrow)

compute right eye $\mathrm{x}$-axis start point $\mathrm{ReX}$.

compute right eye y-axis start point ReY.

step6: Detect Iris from left eye LI.

Detect Iris from right Eye RI.

Detect LI $\mathrm{x}$-axis starting point LIX.

Detect LI y-axis start point LIY.

Detect RI $\mathrm{x}$-axis starting point RIX.

Detect RI y-axis start point RIY.

Compute size of Left iris ALI.//Area of left iris

Compute size of Right Iris RLI.//Area of right iris.

step7: Identify Mouth region Mr.

Compute mouth height mh.

Compute mouth width mw.

Compute lips height lh.

Compute lips X-axis start point LX.
Compute lips y-axis start point LY.

step7: Identify Nose region Nr.

Compute height of nose $\mathrm{Nh}$
$\int \operatorname{Dist}\left(\right.$ RE.TopBrow $\left.-\frac{M h}{2}\right)-L h$

step8: construct feature vector Fv.

FV

\{Lew,Leh,Rew,Reh,LIX,LIY,RIX,RIY,ALI,RLI,Mh,Mw,lh,Lx, LY, LeX,LeY,ReX,ReY, Nh\}.

step9: stop.

\subsection{Eccentric Computation}

The eccentric measure shows the boundary region of the feature and is measured based on the height and width of the feature. For any person the boundary points and area will differ in nature which could be used for person identification. For example the $\mathrm{x}$-axis start point and $\mathrm{y}$-axis start point of eye could be used to compute the eccentric measure and area occupied by the feature eye. We compute such measures for each of the feature identified, so that the area occupied by all the facial features are computed. This may give some valuable results but for accurate identification, we compute eccentric measure of midpoint eccentric feature of both eyes and mouth. The centre point of both iris is identified and $\mathrm{x}$-axis co-ordinates of mouth is identified to compute midpoint eccentric measure.

\section{Algorithm:}

step1: start

step2: read feature set Fv.

step3: compute eccentric measure of left Eye Ele.

Ele $=\int O(F v \cdot L e X, F v \cdot L e Y, F v \cdot L e W, F v . L e h)$

step4: compute eccentric measure of right eye Ere.

Ere $=\int O(F v \cdot \operatorname{ReX}, F v \cdot \operatorname{ReY}, F v \cdot R e W, F v \cdot R e h)$

step5: compute eccentric measure of mouth Em.

$$
\mathrm{Em}=\int O(F v \cdot L X, F v \cdot L Y, F v \cdot l h)
$$

step6: compute midpoint eccentric measure MEM.

$\mathrm{MEM}==\int \emptyset(F v \cdot L I X, F v \cdot L I Y, F v \cdot R I X, R I Y, L X, L Y)$

step7: compute cumulative weight match measure $\mathrm{Cm}$.

$\mathrm{Cm}=\mathrm{MEM} /(($ Ele + Ere + Em $) / \mathrm{Nh})$.

step8: stop

\subsection{Face Recognition}

The person identification is performed by computing the eccentric measures and midpoint eccentric measures of all the facial features. Once all the features and measures are computed then a cumulative weight is computed. The cumulative weight is computed for each sample available in the training set. The sample which has more weight will be selected as the identified person. Unlike other identification methods, the proposed method has used a combined nature of all the facial features which makes face recognition as more sensible. 


\section{RESULTS AND DISCUSSION}

The proposed methodology has been implemented in MATLAB simulation software and performed a many face detection experiments using the described system. The proposed method has been evaluated with RGB Images of human faces and produced satisfactory results. A high face detection rate is obtained. We created a template face set that contains 25 grayscale images of various scales and imaging conditions, for our experiments. As one can see in the pictures below, these templates represent both male and female faces, and people of various ages and races. These faces are also characterized by various orientations and poses, some of them have structural elements, too. The template set can be extended, by adding new faces, but although a large set could improve the detection results, it also produces a high computation complexity.

Our face detection technique is characterized not only by a high detection rate, that is approximately $99 \%$ and indicates a low number of false negatives (missed faces), but also by a low number of false positive (non-facial image regions declared to be faces).

Table 1: Variation of FAR, FRR, RR.

\begin{tabular}{|l|l|l|l|}
\hline $\begin{array}{l}\text { No. of } \\
\text { Samples }\end{array}$ & FRR & FAR & RR in \% \\
\hline 2 & 0.8 & 0.4 & 99.89 \\
\hline 3 & 0.6 & 0.3 & 99.91 \\
\hline 5 & 0.2 & 0.2 & 99.95 \\
\hline
\end{tabular}

The table 1 shows the False rejection rate (FRR), false acceptance rate FAR, recognition rate (RR), values produced by the proposed method at various size of samples in the training set. It shows that if the size of sample increased the ratio of recognition rate is also increases.

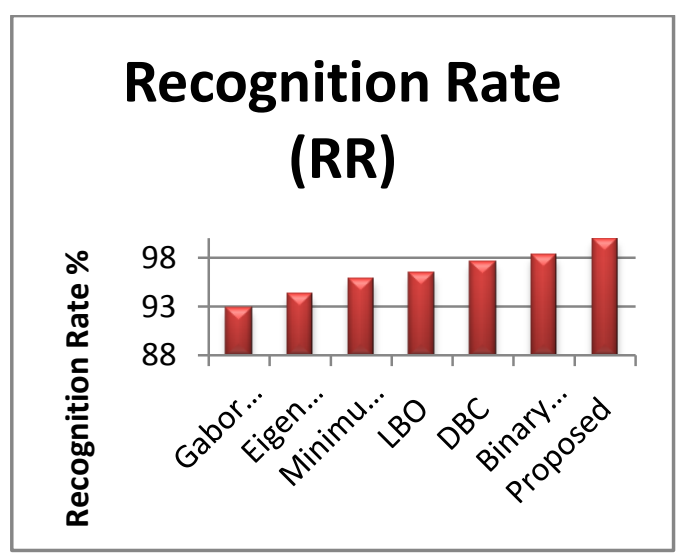

Graph1: Recognition rate achieved.

The graph1 shows the recognition rate achieved by different algorithms at fixed number of samples as training and testing. The methods have been tested with $30 \%$ samples and $70 \%$ as training samples. The proposed method has produced higher rate of recognition than other methods.

\section{Time Complexity}

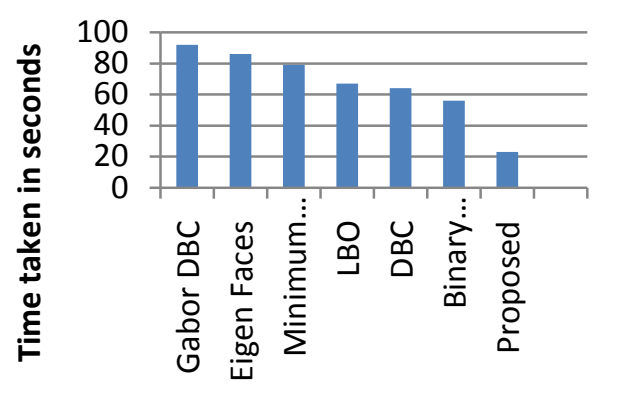

Graph2: shows the time complexity of different methods.

The graph2 shows the time complexity of different methods. It shows that the proposed method has taken less time than other methods. The time complexity has been evaluated with fixed number of test samples and the proposed method has produced higher rate of results.

\section{RESULTS AND DISCUSSION}

We proposed MEN: a multi attribute feature selection method for face recognition. We have identified and extracted all the facial components from face image and for each feature the proposed method computes eccentric measure, midpoint eccentric measure. Based on computed measures a cumulative weight is computed using which person identification is performed. The proposed method has included various features of facial components and produced higher rate of recognition compared to others. Further the recognition rate can be improved by including other co-ordinates of facial components like nose edge and nose tower region features.

\section{REFERENCES}

[1] TahiaFahrinKarim, Md. LushanurRahman, Molla ShahadatHossain Lipu and Faria Sultana, "Face Recognition using PCA-Based Method", IEEE International Conference on AdvancedManagement Science, vol. 3, pp. 158- 162, 2010.

[2] GopaBhaumik, TanwiMallick, Koyel Sinha Chowdhury, GautamSanyal, "Analysis andDetection of Human Faces by using Minimum Distance Classifier for Surveillance", IEEEInternational Conference on Recent Trends in Information, Telecommunication and Computing, pp.265267, 2010.

[3] Gopa Bhaumik, Tanwi Mallick, Koyel Sinha Chowdhury, GautamSanyal, "Analysis andDetection of Human Faces by using Minimum Distance Classifier for Surveillance", IEEEInternational Conference on Recent Trends in Information, Telecommunication and Computing, pp.265267, 2010.

[4] S Sumathi and R Rani HemaMalini, "Face Recognition System to enhance E-Health," IEEEInternational Conference on E-Health Networking, Digital Ecosystems and Technologies, pp. 195-198, 2010.

[5] Haijun Zhang, Q.M.JonathanWu, TommyW.S.Chow, and MingboZhao, "A two-dimensionalNeighborhood Preserving Projection for appearance-based face recognition," ELSEVIERInternational Journal on Pattern Recognition, Vol. 45, Issue 5, pp. 1866-1876,2012. 
[6] Loris Nanni, Alessandra Lumini, and Sheryl Brahnam, "Survey on LBP based texturedescriptors for image classification," ELSEVIER International Journal on Expert Systems withApplications, Vol. 39, Issue 3, pp. 3634-364, 2012.

[7] Francisco A. Pujol and Juan Carlos García, "Computing the Principal Local Binary Patternsfor face recognition using data mining tools," ELSEVIER International Journal on Expert Systemswith Applications, Article in press,2012.
[8] T.Barbu, An Automatic Face Detection System for RGB Images, Int. J. of Computers, Communications \& Control,2011.

[9] Jagadeesh H S1, Suresh Babu K2, and Raja K B2, DBC based Face Recognition using DWT, Signal \& Image Processing : An International Journal (SIPIJ) Vol.3, No.2, April 2012 